\title{
Infection in systemic lupus erythematosus, similarities, and differences with lupus flare
}

\author{
Ju-Yang Jung and Chang-Hee Suh
}

Department of Rheumatology, Ajou University School of Medicine, Suwon, Korea

Received: July 13, 2016

Accepted: February 21, 2017

\section{Correspondence to}

Chang-Hee Suh, M.D.

Department of Rheumatology,

Ajou University School of

Medicine, 164 World cup-ro,

Yeongtong-gu, Suwon 16499 ,

Korea

Tel: +82-31-219-5118

Fax: +82-31-219-5157

E-mail: chsuh@ajou.ac.kr
Systemic lupus erythematosus (SLE) is a chronic autoimmune disease with diverse manifestations, and its pathogenesis is unclear and complicated. Infection and SLE are similar in that they both cause inflammatory reactions in the immune system; however, one functions to protect the body, whereas the other is activated to damage the body. Infection is known as one of the common trigger factors for SLE; there are a number of reports on infectious agents that provoke autoimmune response. Several viruses, bacteria, and protozoa were revealed to cause immune dysfunction by molecular mimicry, epitope spreading, and bystander activation. In contrast, certain pathogens were revealed to protect from immune dysregulation. Infection can be threatening to patients with SLE who have a compromised immune system, and it is regarded as one of the common causes of mortality in SLE. A clinical distinction between infection and lupus flare up is required when patients with SLE present fevers. With a close-up assessment of symptoms and physical examination, C-reactive protein and disease activity markers play a major role in differentiating the different disease conditions. Vaccination is necessary because protection against infection is important in patients with SLE.

Keywords: Lupus erythematosus, systemic; Infection; Fever; Lupus flare; Vaccination

\section{INTRODUCTION}

Systemic lupus erythematosus (SLE) is a heterogeneous autoimmune disease characterized by pathogenic autoantibodies and uncontrolled inflammatory response [1]. It often affects young women during childbearing periods; however, it can also occur at any age in children, the aged, and males [2]. Inflammatory reaction in SLE can target various organs; its symptom manifestations are skin rash including butterfly-shaped malar rash, oral ulcer, arthritis, hemolytic anemia, and nephritis. Moreover, its severity is diverse, from no detectable symptoms or no organ damage to serious and irreversible tissue damage and even death. The organs affected and disease activity are considerably different between individuals and changes as time progresses. For example, it is possible that a patient with lupus nephritis with heavy proteinuria several years ago would now show little symptoms and minimal disease activity. SLE management should be sensitive toward such changes and adjust medications accordingly.

The immunologic abnormalities in patients with SLE have been revealed. The presentation of autoantigens by antigen-presenting cells (APCs), such as macrophages and dendritic cells, to T cells leads to the maturation and release of proinflammatory cytokines. B cells produce autoantibodies forming immune complexes that deposit on tissues and damage them. Upregulation of the type I interferon (IFN) system by stimulated plasmacytoid dendritic cells enhances the maturation or func- 
tion of immune cells and suppresses the differentiation of regulatory $\mathrm{T}$ cells maintaining self-tolerance $[3,4]$.

There have been some long-standing questions about infection and SLE, including whether both diseases are affected by each other, which pathogens cause SLE or are vulnerable to SLE, and how infection and lupus flare can be discriminated if patients with lupus present fevers. It is currently evident that infection affects the development of SLE, and infection occurrence and prevention are also influenced by SLE (Fig. 1). The indications for their close relationship are described here.

\section{INFECTION AS A TRIGGERING FACTOR FOR SLE}

Infectious agents, including viruses, bacteria, parasites, and fungus, are a pivotal factor for induction of autoimmunity. Many pathogens are known to contribute toward abnormal immune responses in genetically susceptible individuals through molecular mimicry, epitope spreading, bystander activation, or other mechanisms [5].

One of the reasons that autoreactive $\mathrm{T}$ and $\mathrm{B}$ cells are triggered by infection is molecular mimicry, in which there is a structural similarity between microbial peptides and self-antigens [6]. The cross-reactivity of microbial peptides and self-peptides allows the expansion of microbial-specific $\mathrm{T}$ cells that can respond to self-pep-

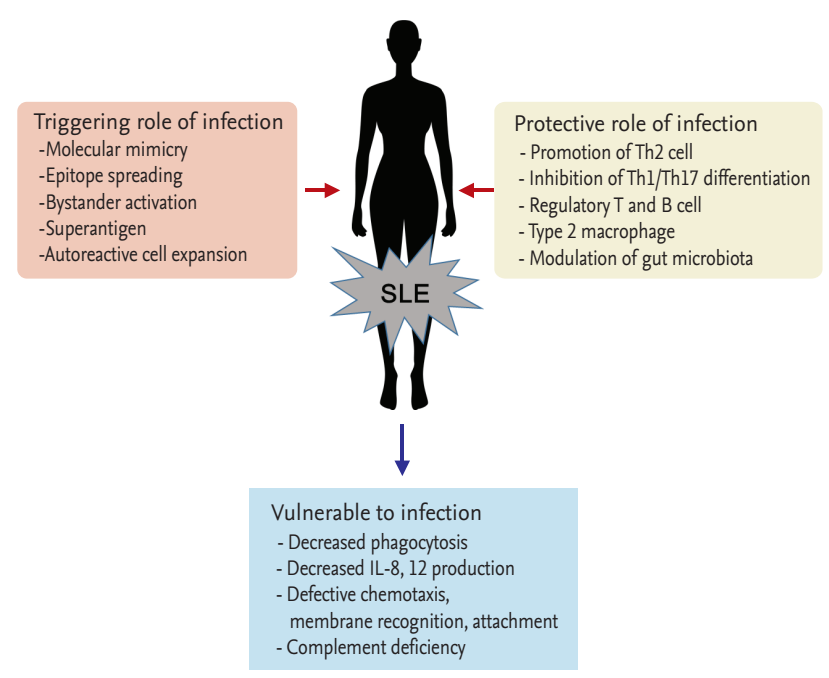

Figure 1. Interaction of infection and systemic lupus erythematosus (SLE). Th, T-helper; IL, interleukin. tides [2]. Pathogens produce superantigens that bind to the variable domain of $\mathrm{T}$ cell receptors and various major histocompatibility complex class II molecules. Subsequently, a large number of $\mathrm{T}$ cells with different antigenic specificities are activated and lead to autoimmune reactions. The presentation of self-antigens by APCs cause the priming of a number of $\mathrm{T}$ cells by epitope spreading, overprocessing, and overpresentation of self-antigens. As T cells are activated, cytokine production increases autoreactive or memory $\mathrm{T}$ cell expansion via a process called bystander activation. In particular, viral infections such as hepatitis $\mathrm{C}$ infections target $\mathrm{B}$ lymphocytes and induce them to produce antibodies, leading to the formation of immune complexes. Moreover, the impaired clearance of apoptotic cells results in the insufficient removal of infectious agents $[7,8]$. Nuclear materials derived from apoptotic cells are exposed to the immune system, causing the production of autoantibodies or activation of autoreactive lymphocytes. Pathogens including bacteria and viruses interact with intracellular toll-like receptors (TLRs) through intracellular signaling pathways, leading to the expression of type I IFN genes. Type I IFN signatures are major pathological indicators of autoimmune diseases such as SLE, Sjogren's syndrome, and polymyositis [9].

\section{Viral infection}

Epstein-Barr virus (EBV) or human herpesvirus 4 is a ubiquitous virus that infects about $95 \%$ of the world's population [10]. EBV infection is known to occur during childhood without any symptoms; however, it causes infectious mononucleosis in adults and is characterized by skin rash, arthralgia, renal disorders, cytopenia, pharyngitis, and lymphadenopathy. Many studies have found that EBV infection is related to systemic autoimmunity [10]. SLE patients had higher titers of anti-EBV antibodies, and the prevalence of EBV infection in patients with SLE was higher than in the healthy population [11-13]. It was revealed that EBV showed increased replication with activation of the lytic cycle in patients with SLE, and EBV-infected B cells might become resistant to apoptosis. Moreover, EBV infection causes T cells to produce more IFNs that are pivotal proinflammatory cytokines for systemic autoimmunity. Components of the EBV protein, including Epstein-Barr nuclear antigen-1, showed similarity to autoantigens in SLE; thereby, sug- 
gesting molecular mimicry as a potential mechanism of antibody cross-reactivity and disease pathogenesis [14]. In a recent study, antibodies of early EBV infection were detected at higher levels in patients with SLE than in healthy controls, and the reactivation of EBV seemed to correlate with lymphopenia [15].

Human endogenous retroviruses (HERVs) were integrated into the human genome about 30 to 40 million years ago, and the endogenous retroviral sequence (ERS) encodes self-antigens that contribute toward immunological tolerance breakdown $[16,17]$. Several haplotypes of human ERS, such as human T cell lymphotropic virus 1-related endogenous sequence (HRES-1), were revealed to affect the development of SLE [18]. HERV-E 4-1 mRNA expression was higher than healthy controls and correlated with disease activity in SLE, and SLE CD $4+\mathrm{T}$ cells had decreased methylation levels of the long terminal repeats in HERV-E 4-1 [19].

Parvovirus B19 infection is generally asymptomatic or self-limited; however, it can present a systemic immune response mimicking SLE flare, including arthritis and skin rash [20]. Parvovirus B19 infection induces $\mathrm{T}$ cell activation through molecular mimicry, leading to tissue damage and cell necrosis. Ku8o, a lupus autoantigen that consists of nuclear DNA-binding proteins, functions as a specific B19 capsid-binding surface protein. The disease-related epitope binds with viral particles and activates the immune system.

Cytomegalovirus (CMV) is a member of the herpesvirus family, and it is commonly transmitted during childhood with self-limiting, or no symptoms [21]. CMV infection typically presents severe organ-specific complications in the immunosuppressed host. Although the relationship between EBV or parvovirus B19 infection and SLE seems consistent, the correlation between CMV infection and SLE development has been controversial $[12,22]$. Nevertheless, in recent studies, the detection of specific human CMV strains and CMV antibodies were higher in patients with SLE than in healthy controls $[15,23]$.

\section{Bacterial infection}

Bacterial lipopolysaccharides or nucleic acid-containing immune complexes engage and deteriorate the immune system [14]. Bacterial products that are called pathogen-associated molecular patterns bind to TLRs or other receptors on APCs, B cells, and T cells. Their interactions activate immune cells to produce proinflammatory cytokines that lead to innate immunity activation. TLR ligands stimulate plasmacytoid dendritic cells to produce IFN, resulting in release of autoantibodies. In addition, tissue injuries by bacteria produce cellular debris such as nuclear components that can act as autoantigens. The release of cholera toxin B by Vibrio cholera was revealed to produce autoantibodies and promote glomerulonephritis in lupus-prone mice by enhancing lipid raft aggregation in T cells [24].

\section{INFECTION AS A PROTECTOR AGAINST SLE}

Infection by certain parasites or bacteria has been known to confer protection against autoimmune or allergic diseases; this is known as the "hygiene hypothesis" [25-27]. This hypothesis was introduced because the prevalence of allergic and autoimmune diseases showed a steep increase over the past few decades; however, background studies have not been conducted until now. The intricate mechanism involved includes promotion of T-helper 2 (Th2) and inhibition of Th1/Th17 differentiation, amplication of T-regulatory (Treg) and B-regulatory (Breg) cells and type 2 macrophages, orientation of dendritic cells toward a tolerogenic phenotype, downregulation of type 2 innate lymphoid cells, and modulation of gut microbiota. These beneficial microbes have been called 'old friends,' which means they exert an immunomodulatory and immunosuppressive action on the mammalian immune system [28].

Before the hygiene hypothesis was introduced, the protective role of infection against autoimmune disease development has been investigated. In lupus-prone mice, persistent infection with lactate dehydrogenase virus results in lower autoantibodies and protection from nephritis [29]. The immune response generated by malarial infection (Plasmodium falciparum) was shown to confer protection against the development of SLE because the gene related to malaria protection is an SLE-susceptible gene [30,31]. Malaria parasite infection of lupus mice controls B cell autoreactivity and confers protection against histopathological alterations and lupus severity [32,33]. Toxoplasma gondii infection had a protective role in preventing the development of 
autoimmune renal disorder in lupus-prone mice [34]. A glycoprotein secreted by parasitic helminths prevented nephritis in lupus mice model via reduction of antinuclear antibody (ANA) production and immune complex deposition [35]. Helicobacter pylori seronegativity was associated with the development of SLE in African-American women [36]. In some studies, the prevalence of hepatitis B virus (HBV) infection in patients with SLE was lower than that in non-SLE controls, thus revealing a protective role of $\mathrm{HBV}$ against SLE [37,38].

\section{INFECTION AS A VULNERABLE POINT IN SLE}

Infection is common in SLE and accounts for $25 \%$ to $50 \%$ of overall mortality $[39,40]$. Half of the patients with SLE experience severe infection, and more than $20 \%$ of hospitalizations occur from infections [41]. When superfluous immune reactivation damages diverse organs with constitutional symptoms, defense mechanisms against bacteria, viruses, or fungi become impaired in SLE. Decreased phagocytosis, reduced production of interleukin 8 (IL-8) and IL12 by polymorph nuclear cells, complement deficiency, and defective chemotaxis, membrane recognition, and attachment to microorganisms are known to predispose SLE patients to infection [42].

Bacterial infections are commonly reported in SLE and affect diverse organs, including the respiratory tract, urinary tract, and skin [41]. Streptococcus pneumonia is a common cause of respiratory infection. Staphylococcus aureus causes skin, soft tissue, bone, and joint infections and bacteremia. Escherichia coli is the most common cause of urinary tract infection; Klebsilla and Pseudomonas spp. also cause urinary tract infections.

Among the viral infections, that by varicella-zoster virus (VZV) is the most frequent in SLE patients, and can develop into herpes zoster [43]. The concomitant use of corticosteroids and immunosuppressive agents agents are regarded as triggering factors for VZV infection, and this infection is more frequent among patients with no or low SLE disease activity [44]. The prevalence of herpes zoster in SLE has increased, and risk of herpes zoster is associated with lymphopenia, increased use of corticosteroids, and most types of immunosuppressive therapy [45-47]. A poor cellular immunity, including decreased
IFN- $\gamma$ releasing cells and $\mathrm{CD}_{4}+\mathrm{T}$ cells in response to $\mathrm{VZV}$, is correlated with VZV infection and SLE [48]. CMV infection can be presented as CMV retinitis, CMV colitis, or CMV pneumonitis in patients with SLE, and may result in a systemic inflammation mimicking SLE [49]. When human papillomavirus (HPV) infection was assessed among patients with SLE, high-risk and multiple HPV infections were more frequent in SLE patients than in controls, and such a trend increased in patients with major organ involvement or immunosuppressant therapies [50,51]. In addition, the prevalence of abnormal squamous epithelial lesions was increased in patients with SLE with HPV infection [52].

The risk factors for infection in patients with SLE are disease activity, such as anti-double-stranded DNA (dsDNA) titer, low complement levels, nephritis and leucopenia, corticosteroids treatment at a prednisolone equivalent dose of 7.5 to $10 \mathrm{mg} /$ day, corticosteroid pulse therapy, and high-dose regimen of cyclophosphamide [53-56]. Several studies showed that most infections, from mild to severe, can be associated with the Systemic Lupus Erythematosus Disease Activity Index (SLEDAI) score that assesses the current disease activity of SLE. Cyclophosphamide is an alkylating agent used in severe manifestations of SLE such as lupus nephritis and mesenteric vasculitis, and it can strongly suppress the immune system. A higher dose of cyclophosphamide was associated with a higher prevalence of infection, and leucopenia after cyclophosphamide therapy also affects infection [57]. It is well known that corticosteroids increase susceptibility to infections, and their immunosuppressive effect is stronger when higher doses are used for a longer period [58]. However, especially in Korean patients with SLE who are not on glucocorticoids, increased SLEDAI, anemia, and active urinary sediment were associated with infection [56].

Tuberculosis (TB) caused by Mycobacterium tuberculosis is regarded as a serious infection that results in pulmonary damage in SLE, and its risk and prevalence are much higher in SLE when combined with a defective immune system and use of immunosuppressive drugs [59,6o]. Use of glucocorticoids, arthritis, pleuritis, vasculitis, nephritis, organic brain syndrome, previous TB, and duration of SLE have been shown to be factors associated with TB in SLE [61,62]. Incidences of TB, extrapulmonary T'B rates, and relapse rates were higher 
in Korean patients with SLE than in Korean patients with rheumatoid arthritis [63]. Before starting any potent immunosuppressive therapy, screening for latent TB should be performed in an endemic area like Korea. If a result is positive, treatment for latent TB would be needed. As a screening tool for latent TB, the tuberculin skin test shows a false-positive result owing to the mandatory BCG vaccination in Korea. Therefore, the interferon $\gamma$ release assay (IGRA) has a critical role in screening latent TB among Korean patients with rheumatic diseases. Recently we reported that IGRA was in agreement with skin test results in patients with SLE, and SLEDAI score and glucocorticoid dose were associated with the indeterminate results of IGRA [64].

\section{DIFFERENTIATION BETWEEN INFECTION AND LUPUS FLARE UP DURING FEVER}

Fever is a typical symptom not only of infection but also of SLE flare up. When patients with SLE present fevers, physicians will need to determine the cause of the fever. Differentiation is important for further symptom management because each of the different cause of fever, infection or lupus flare up, requires directly opposite treatment from each other. If patients have an infection-related fever, they need antimicrobial agents, whereas if patients have an SLE-related fever, they need immunosuppressive therapy including steroids. Generally, infection is developed from a definite origin, and immune-related symptoms such as arthritis and skin rash are presented with fever in active SLE. If an evident source of infection exists, designing a treatment plan is easy; however, patients with fever typically show no infection source or an ambiguous disease manifestation. For a detailed and exact analysis of infection and disease manifestations, several laboratory findings are helpful to differentiate between the two conditions (Table 1). Since C-reactive protein (CRP) is unresponsive to non-infectious inflammation in SLE, an elevated level of CRP suggests a combined infection in patients with SLE $[56,65,66]$. In our recent study on Korean patients with lupus, CRP levels higher than $1.35 \mathrm{mg} / \mathrm{dL}$ indicated the presence of an infection with 100\% sensitivity and $90 \%$ specificity [66]. Leukocyte count is elevated in SLE with infection, and remains unchanged or decreased in SLE with flare [67]. Complement levels and anti-dsDNA antibody titer are changed only in SLE with flare, but the changes are usually not considerable at already abnormal levels. Overall, CRP is the most critical marker in differentiating between infection and disease flare up in SLE. In a recent study, fever duration, anti-dsDNA antibody titers, and CRP were regarded as the most reliable markers to distinguish between infection and SLE activity; a flare risk predictive calculator using these markers was proposed [68]. In addition, neutrophil CD64 expression (or Fcy receptor I), percentage of circulating $\mathrm{CD}_{27}$ high plasma cells, S10oA8/A9 protein, and procalcitonin have been suggested and studied as new promising biomarkers to assist clinical decision-making [69,70].

\section{INFECTION AS A TARGET OF VACCINE IN SLE}

Vaccination is considered an essential method to prevent infections or reduce complications in SLE, although

Table 1. Comparison of biomarker changes between infection and systemic lupus erythematosus flare up

\begin{tabular}{|c|c|c|c|}
\hline Biomarkers & Infection & SLE flare up & References \\
\hline Leukocyte count & Elevated & Reduced & {$[65,67,68]$} \\
\hline ESR & Elevated & Elevated & {$[56,66,68]$} \\
\hline CRP & Elevated & $\begin{array}{l}\text { No change or } \\
\text { minimally elevated }\end{array}$ & {$[56,65,66,68]$} \\
\hline $\mathrm{C}_{3}$ and $\mathrm{C}_{4}, \mathrm{CH}_{5} \mathrm{O}$ & No change or elevated & Reduced & {$[65,66,68]$} \\
\hline Anti-dsDNA antibody & No change & Elevated & {$[65,66,68]$} \\
\hline IL-6, IL-10 and IFN- $\gamma$, S10o/A9, procalcitonin & Elevated & Elevated & {$[65,66,69,70]$} \\
\hline
\end{tabular}

SLE, systemic lupus erythematosus; ESR, erythrocyte sedimentation rate; CRP, C-reactive protein; $\mathrm{C}_{3}$, complement 3; 4 , complement 4; dsDNA, double-strand DNA; IL, interleukin; IFN, interferon. 
Table 2. The guidelines for vaccination in systemic lupus erythematosus

\begin{tabular}{ll}
\hline Condition & Remission or stable status \\
& Without immunosuppressive agents (cyclophosphamide, methotrexate) \\
& Taking glucocorticoids (less than $20 \mathrm{mg} /$ day of prednisolone or equivalent) \\
Vaccine & Yearly influenza \\
& 23-Valent polysaccharide pneumococcal agent \\
& Anti-tetanus agent \\
& HAV and HBV for high-risk group \\
& HPV for female patients older than 25 years \\
Avoidance & Live vaccination with immunosuppressive therapy \\
\hline
\end{tabular}

HAV, hepatitis A virus; HBV, hepatitis B virus; HPV, human papilloma virus.

the clinical effects are not prominent [71]. According to the European League Against Rheumatism recommendations for vaccination in adult patients with autoimmune inflammatory diseases, vaccination status needs to be determined during the initial investigation, and it includes Haemophilus influenzae b, hepatitis A virus, $\mathrm{HBV}, \mathrm{HPV}$, influenza, Neisseria meningitidis, rubella for women of childbearing age, S. pneumonia, and tetanus toxoid [72]. Vaccinations should be administered during stable disease and before B cell depletion therapy (Table 2). However, vaccines are thought to trigger immune system activation because they stimulate an antigen-specific immune response similarly to the molecular mimicry described previously [73]. There were several reports on SLE development or flare up after vaccination, and numerous cases of SLE in relation with HBV vaccination have been reported during the 1990s. Their causality was not clearly identified, but the temporal connection of vaccination with symptom development was consistent. On the contrary, it has been observed that the weaker immunologic response presents a protective function against pathogens in patients with SLE than in healthy controls after vaccination $[74,75]$. The safety of vaccines in patients with SLE has been demonstrated; a recent case-control study showed that immunization is not associated with SLE development [76-78]. Therefore, vaccinations against diseases including HBV, HPV, pneumococcal, and influenza are recommended for patients with SLE in remission period. An inactive live vaccine such as zoster vaccine should be avoided when the patients are on immunosuppressive drugs such as cyclophosphamide, methotrexate, and corticosteroids (over $20 \mathrm{mg} /$ day of prednisolone or equivalent).

\section{CONCLUSIONS}

Among the environmental factors causing or aggravating SLE, infection is most well described; however, how it works or who is vulnerable to it remains unclear. Currently, several viruses including EBV, parvovirus B19, and retrovirus may contribute to SLE development. In addition, certain pathogens such as T. gondii, Plasmodi$u m$, and $H$. pylori may play a role in preventing autoimmune inflammation.

It is evident that patients with SLE are vulnerable to infection because of the nature of the disease and symptom management practices that suppress one's immunity. When patients with SLE present fevers, SLE-related manifestations and disease activity markers indicate an SLE flare up, and increased CRP and leukocyte count suggest a combined infection. Despite its concerns in SLE, vaccination is indispensable for patients with this disease.

\section{Conflict of Interest}

No potential conflict of interest relevant to this article was reported.

\section{Acknowledgments}

This research was supported by a grant of the Korea Health Technology R\&D Project through the Korea Health Industry Development Institute (KHIDI), funded by the Ministry of Health and Welfare, Republic of Korea (grant number: HI16Co992). 


\section{REFRENCES}

1. Lisnevskaia L, Murphy G, Isenberg D. Systemic lupus erythematosus. Lancet 2014;384:1878-1888.

2. Hwang J, Lee J, Ahn JK, Park EJ, Cha HS, Koh EM. Clinical characteristics of male and female Korean patients with systemic lupus erythematosus: a comparative study. Korean J Intern Med 2015;30:242-249.

3. Hagberg N, Ronnblom L. Systemic lupus erythematosus: a disease with a dysregulated type I interferon system. Scand J Immunol 2015;82:199-207.

4. Ohl K, Tenbrock K. Regulatory T cells in systemic lupus erythematosus. Eur J Immunol 2015;45:344-355.

5. Esposito S, Bosis S, Semino M, Rigante D. Infections and systemic lupus erythematosus. Eur J Clin Microbiol Infect Dis 2014;33:1467-1475.

6. Wucherpfennig KW. Mechanisms for the induction of autoimmunity by infectious agents. J Clin Invest 2001;108:1097-1104.

7. Jung JY, Suh CH. Incomplete clearance of apoptotic cells in systemic lupus erythematosus: pathogenic role and potential biomarker. Int J Rheum Dis 2015;18:294-303.

8. Munoz LE, van Bavel C, Franz S, Berden J, Herrmann M, van der Vlag J. Apoptosis in the pathogenesis of systemic lupus erythematosus. Lupus 2008;17:371-375.

9. Koutouzov S, Mathian A, Dalloul A. Type-I interferons and systemic lupus erythematosus. Autoimmun Rev 2006;5:554-562.

10. Draborg AH, Duus K, Houen G. Epstein-Barr virus and systemic lupus erythematosus. Clin Dev Immunol 2012;2012:370516.

11. James JA, Kaufman KM, Farris AD, Taylor-Albert E, Lehman TJ, Harley JB. An increased prevalence of Epstein-Barr virus infection in young patients suggests a possible etiology for systemic lupus erythematosus. J Clin Invest 1997;100:3019-3026.

12. Perez-Mercado AE, Vila-Perez S. Cytomegalovirus as a trigger for systemic lupus erythematosus. J Clin Rheumatol 2010;16:335-337.

13. Barzilai O, Sherer Y, Ram M, Izhaky D, Anaya JM, Shoenfeld Y. Epstein-Barr virus and cytomegalovirus in autoimmune diseases: are they truly notorious? A preliminary report. Ann N Y Acad Sci 2007;1108:567-577.

14. Francis L, Perl A. Infection in systemic lupus erythematosus: friend or foe? Int J Clin Rheumtol 2010;5:59-74.

15. Rasmussen NS, Draborg AH, Nielsen CT, Jacobsen S,
Houen G. Antibodies to early EBV, CMV, and HHV6 antigens in systemic lupus erythematosus patients. Scand J Rheumatol 2015;44:143-149.

16. Herrmann M, Hagenhofer M, Kalden JR. Retroviruses and systemic lupus erythematosus. Immunol Rev 1996;152:145-156.

17. Tugnet N, Rylance P, Roden D, Trela M, Nelson P. Human endogenous retroviruses (HERVs) and autoimmune rheumatic disease: is there a link? Open Rheumatol J 2013;7:13-21.

18. Pullmann R Jr, Bonilla E, Phillips PE, Middleton FA, Perl A. Haplotypes of the HRES-1 endogenous retrovirus are associated with development and disease manifestations of systemic lupus erythematosus. Arthritis Rheum 2008;58:532-540.

19. Wu Z, Mei X, Zhao D, et al. DNA methylation modulates HERV-E expression in CD4+ T cells from systemic lupus erythematosus patients. J Dermatol Sci 2015;77:110-116.

20. Aslanidis S, Pyrpasopoulou A, Kontotasios K, Doumas $\mathrm{S}$, Zamboulis C. Parvovirus B19 infection and systemic lupus erythematosus: activation of an aberrant pathway? Eur J Intern Med 2008;19:314-318.

21. Gandhi MK, Khanna R. Human cytomegalovirus: clinical aspects, immune regulation, and emerging treatments. Lancet Infect Dis 2004;4:725-738.

22. Mohamed AE, Hasen AM, Mohammed GF, Elmaraghy NN. Real-time PCR of cytomegalovirus and Epstein-Barr virus in adult Egyptian patients with systemic lupus erythematosus. Int J Rheum Dis 2015;18:452-458.

23. Chen J, Zhang H, Chen P, et al. Correlation between systemic lupus erythematosus and cytomegalovirus infection detected by different methods. Clin Rheumatol 2015;34:691-698.

24. Deng GM, Tsokos GC. Cholera toxin B accelerates disease progression in lupus-prone mice by promoting lipid raft aggregation. J Immunol 2008;181:4019-4026.

25. Versini M, Jeandel PY, Bashi T, Bizzaro G, Blank M, Shoenfeld Y. Unraveling the hygiene hypothesis of helminthes and autoimmunity: origins, pathophysiology, and clinical applications. BMC Med 2015;13:81.

26. Vercelli D. Mechanisms of the hygiene hypothesis: molecular and otherwise. Curr Opin Immunol 2006;18:733737.

27. Weinstock JV. Autoimmunity: the worm returns. Nature 2012;491:183-185.

28. Rook GA. Hygiene hypothesis and autoimmune diseases. 
Clin Rev Allergy Immunol 2012;42:5-15.

29. Oldstone MB, Dixon FJ. Inhibition of antibodies to nuclear antigen and to DNA in New Zealand mice infected with lactate dehydrogenase virus. Science 1972;175:784-786.

30. Clatworthy MR, Willcocks L, Urban B, et al. Systemic lupus erythematosus-associated defects in the inhibitory receptor FcgammaRIIb reduce susceptibility to malaria. Proc Natl Acad Sci U S A 2007;104:7169-7174.

31. Willcocks LC, Carr EJ, Niederer HA, et al. A defunctioning polymorphism in FCGR2B is associated with protection against malaria but susceptibility to systemic lupus erythematosus. Proc Natl Acad Sci U S A 2010;107:7881-7885.

32. Badr G, Sayed A, Abdel-Maksoud MA, et al. Infection of female BWF1 lupus mice with malaria parasite attenuates B cell autoreactivity by modulating the CXCL12/CXCR4 axis and its downstream signals $\mathrm{PI}_{3} \mathrm{~K} / \mathrm{AKT}$, NFkappaB and ERK. PLoS One 2015;10:e0125340.

33. Abdel-Maksoud MA, Abdel-Ghaffar FA, El-Amir A, AlQuraishy S, Badr G. Infection with plasmodium chabaudi diminishes plasma immune complexes and ameliorates the histopathological alterations in different organs of female BWF1 lupus mice. Eur Rev Med Pharmacol Sci 2016;20:733-744.

34. Chen M, Aosai F, Norose K, et al. Toxoplasma gondii infection inhibits the development of lupus-like syndrome in autoimmune (New Zealand Black x New Zealand White) F1 mice. Int Immunol 2004;16:937-946.

35. Rodgers DT, McGrath MA, Pineda MA, et al. The parasitic worm product ES-62 targets myeloid differentiation factor 88-dependent effector mechanisms to suppress antinuclear antibody production and proteinuria in MRL/lpr mice. Arthritis Rheumatol 2015;67:1023-1035.

36. Sawalha AH, Schmid WR, Binder SR, Bacino DK, Harley JB. Association between systemic lupus erythematosus and Helicobacter pylori seronegativity. J Rheumatol 2004;31:1546-1550.

37. Zhao J, Qiu M, Li M, Lu C, Gu J. Low prevalence of hepatitis B virus infection in patients with systemic lupus erythematosus in southern China. Rheumatol Int 2010;30:1565-1570.

38. Ram M, Anaya JM, Barzilai O, et al. The putative protective role of hepatitis B virus (HBV) infection from autoimmune disorders. Autoimmun Rev 2008;7:621-625.

39. Petri M. Infection in systemic lupus erythematosus. Rheum Dis Clin North Am 1998;24:423-456.

40. Wang Z, Wang Y, Zhu R, et al. Long-term survival and death causes of systemic lupus erythematosus in China: a systemic review of observational studies. Medicine (Baltimore) 2015;94:e794.

41. Goldblatt F, Chambers S, Rahman A, Isenberg DA. Serious infections in British patients with systemic lupus erythematosus: hospitalisations and mortality. Lupus 2009;18:682-689.

42. Cuchacovich R, Gedalia A. Pathophysiology and clinical spectrum of infections in systemic lupus erythematosus. Rheum Dis Clin North Am 2009;35:75-93.

43. Gladman DD, Hussain F, Ibanez D, Urowitz MB. The nature and outcome of infection in systemic lupus erythematosus. Lupus 2002;11:234-239.

44. Borba EF, Ribeiro AC, Martin P, Costa LP, Guedes LK, Bonfa E. Incidence, risk factors, and outcome of Herpes zoster in systemic lupus erythematosus. J Clin Rheumatol 2010;16:119-122.

45. Hu SC, Yen FL, Wang TN, Lin YC, Lin CL, Chen GS. Immunosuppressive medication use and risk of herpes zoster (HZ) in patients with systemic lupus erythematosus (SLE): a nationwide case-control study. J Am Acad Dermatol 2016;75:49-58.

46. Murray SG, Schmajuk G, Trupin L, et al. National lupus hospitalization trends reveal rising rates of herpes zoster and declines in pneumocystis pneumonia. PLoS One 2016;11:e0144918.

47. Ferreira JC, Marques HH, Ferriani MP, et al. Herpes zoster infection in childhood-onset systemic lupus erythematosus patients: a large multicenter study. Lupus 2016;25:754-759.

48. Rondaan C, de Haan A, Horst G, et al. Altered cellular and humoral immunity to varicella-zoster virus in patients with autoimmune diseases. Arthritis Rheumatol 2014;66:3122-3128.

49. Ramos-Casals M, Cuadrado MJ, Alba P, et al. Acute viral infections in patients with systemic lupus erythematosus: description of 23 cases and review of the literature. Medicine (Baltimore) 2008;87:311-318.

50. Tam LS, Chan AY, Chan PK, Chang AR, Li EK. Increased prevalence of squamous intraepithelial lesions in systemic lupus erythematosus: association with human papillomavirus infection. Arthritis Rheum 2004;50:3619-3625.

51. Lee YH, Choe JY, Park SH, et al. Prevalence of human papilloma virus infections and cervical cytological abnormalities among Korean women with systemic lupus erythematosus. J Korean Med Sci 2010;25:1431-1437. 
52. Nath R, Mant C, Luxton J, et al. High risk of human papillomavirus type 16 infections and of development of cervical squamous intraepithelial lesions in systemic lupus erythematosus patients. Arthritis Rheum 2007;57:619-625.

53. Duffy KN, Duffy CM, Gladman DD. Infection and disease activity in systemic lupus erythematosus: a review of hospitalized patients. J Rheumatol 1991;18:1180-1184.

54. Bosch X, Guilabert A, Pallares L, et al. Infections in systemic lupus erythematosus: a prospective and controlled study of 110 patients. Lupus 2006;15:584-589.

55. Jeong SJ, Choi H, Lee HS, et al. Incidence and risk factors of infection in a single cohort of 110 adults with systemic lupus erythematosus. Scand J Infect Dis 2009;41:268-274.

56. Suh CH, Jeong YS, Park HC, et al. Risk factors for infection and role of C-reactive protein in Korean patients with systemic lupus erythematosus. Clin Exp Rheumatol 2001;19:191-194.

57. Houssiau FA, Vasconcelos C, D'Cruz D, et al. Immunosuppressive therapy in lupus nephritis: the Euro-Lupus Nephritis Trial, a randomized trial of low-dose versus high-dose intravenous cyclophosphamide. Arthritis Rheum 2002;46:2121-2131.

58. Cutolo M, Seriolo B, Pizzorni C, et al. Use of glucocorticoids and risk of infections. Autoimmun Rev 2008;8:153155 .

59. Feng PH, Tan TH. Tuberculosis in patients with systemic lupus erythematosus. Ann Rheum Dis 1982;41:11-14.

6o. Kang I, Park SH. Infectious complications in SLE after immunosuppressive therapies. Curr Opin Rheumatol 2003;15:528-534.

61. Chang YS, Liu CJ, Ou SM, et al. Tuberculosis infection in primary Sjogren's syndrome: a nationwide population-based study. Clin Rheumatol 2014;33:377-383.

62. Prabu V, Agrawal S. Systemic lupus erythematosus and tuberculosis: a review of complex interactions of complicated diseases. J Postgrad Med 2010;56:244-250.

63. Yun JE, Lee SW, Kim TH, et al. The incidence and clinical characteristics of Mycobacterium tuberculosis infection among systemic lupus erythematosus and rheumatoid arthritis patients in Korea. Clin Exp Rheumatol 2002;20:127-132.

64. Cho H, Kim YW, Suh CH, et al. Concordance between the tuberculin skin test and interferon gamma release assay (IGRA) for diagnosing latent tuberculosis infection in patients with systemic lupus erythematosus and patient characteristics associated with an indeterminate IGRA.
Lupus 2016;25:1341-1348.

65. Suh CH, Chun HY, Ye YM, Park HS. Unresponsiveness of C-reactive protein in the non-infectious inflammation of systemic lupus erythematosus is associated with interleukin 6. Clin Immunol 2006;119:291-296.

66. Kim HA, Jeon JY, An JM, Koh BR, Suh CH. C-reactive protein is a more sensitive and specific marker for diagnosing bacterial infections in systemic lupus erythematosus compared to S1ooA8/A9 and procalcitonin. J Rheumatol 2012;39:728-734.

67. Kim HA, Jeon JY, Choi GS, et al. The antichromatin antibodies can be useful as a diagnostic tool and disease activity marker of systemic lupus erythematosus in Koreans. Clin Immunol 2008;128:277-283.

68. Beca S, Rodriguez-Pinto I, Alba MA, Cervera R, Espinosa G. Development and validation of a risk calculator to differentiate flares from infections in systemic lupus erythematosus patients with fever. Autoimmun Rev 2015;14:586593.

69. Lacki JK, Leszczynski P, Kelemen J, Muller W, Mackiewicz $\mathrm{SH}$. Cytokine concentration in serum of lupus erythematosus patients: the effect on acute phase response. J Med 1997;28:99-107.

70. Sciascia S, Ceberio L, Garcia-Fernandez C, Roccatello D, Karim Y, Cuadrado MJ. Systemic lupus erythematosus and infections: clinical importance of conventional and upcoming biomarkers. Autoimmun Rev 2012;12:157-163.

71. Pasoto SG, Ribeiro AC, Bonfa E. Update on infections and vaccinations in systemic lupus erythematosus and Sjogren's syndrome. Curr Opin Rheumatol 2014;26:528537.

72. van Assen S, Agmon-Levin N, Elkayam O, et al. EULAR recommendations for vaccination in adult patients with autoimmune inflammatory rheumatic diseases. Ann Rheum Dis 2011;70:414-422.

73. Aron-Maor A, Shoenfeld Y. Vaccination and systemic lupus erythematosus: the bidirectional dilemmas. Lupus 2001;10:237-240.

74. Wiesik-Szewczyk E, Romanowska M, Mielnik P, et al. Anti-influenza vaccination in systemic lupus erythematosus patients: an analysis of specific humoral response and vaccination safety. Clin Rheumatol 2010;29:605-613.

75. Elkayam O, Paran D, Caspi D, et al. Immunogenicity and safety of pneumococcal vaccination in patients with rheumatoid arthritis or systemic lupus erythematosus. Clin Infect Dis 2002;34:147-153. 
76. Grimaldi-Bensouda L, Le Guern V, Kone-Paut I, et al. The risk of systemic lupus erythematosus associated with vaccines: an international case-control study. Arthritis Rheumatol 2014;66:1559-1567.

77. Dell' Era L, Esposito S, Corona F, Principi N. Vaccination of children and adolescents with rheumatic diseases.
Rheumatology (Oxford) 2011;50:1358-1365.

78. Elkayam O, Paran D, Burke M, et al. Pneumococcal vaccination of patients with systemic lupus erythematosus: effects on generation of autoantibodies. Autoimmunity 2005;38:493-496. 